\title{
Design of a Robot for the 2019 NASA Robotic Mining Competition
}

\author{
Matthew Braccio ${ }^{1}$ and Philip Voglewede ${ }^{2}$

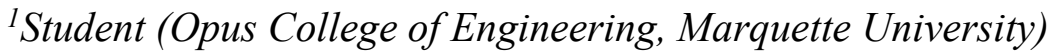 \\ Milwaukee, WI, USA \\ ${ }^{2}$ Professor (Mechanical Engineering, Marquette University) Milwaukee, \\ WI, USA
}

\begin{abstract}
The viability of extracting water on both the Moon and Mars is a focus of NASA as they explore the idea of In-Situ Resource Utilization (ISRU). Marquette Aerospace and Robotic Systems (MARS) is participating in the 2019 NASA Robotic Mining Competition (RMC), a competition driven by using the systems engineering process to design, build, and test a robot capable of autonomously mining icy-regolith simulant in an off world terrain to simulate an ISRU mining mission (NASA, 2018). The team used systems engineering to guide technical management, design, and operations of the robot. With feedback from the team's faculty advisor and industry sponsors, the team utilized multiple systems engineering approaches. No single systems engineering process was selected, rather the team drew from multiple reference texts. The proposed design satisfies the requirements outlined in the 2019 NASA RMC Rules and Rubrics and placed $6^{\text {th }}$ out of 50 in the competition.
\end{abstract}

\section{Introduction}

The 2019 NASA Robotic Mining Competition was Marquette University's first entry into such a competition, and as such the team was tasked with building a design from the ground up. The competition rubrics laid out 3 major sections for scoring points: Design, On-Site Mining, and Outreach, each worth one third of the points for the competition. The design portion, outlined in this paper, required a final report documenting the robot design from a systems-engineering perspective, as well as periodic updates in the form of project plans and schedules demonstrating progress in the competition. The on-site mining portion of the competition required a functioning build of the design that was placed in a simulated Martian environment to test its effectiveness. The outreach portion of the competition involved creating outreach initiatives to encourage STEM education, particularly in underrepresented and underserved communities. From the final score of these three portions of the competition, Marquette University placed $6^{\text {th }}$ out of 50 overall.

\subsection{Problem definition}

As the Orion mission to Mars prepares for its first flight in the coming years, NASA hosted their tenth annual Robotic Mining Competition. This competition provides university students in the United States the opportunity to design and build robots to collect a necessary resource on Mars: water. The scope of the RMC focuses on the collection of water in the form of silica rock deposits, known as BP-1. These robots have the potential to become the supply chain for the first Martian colonies.

\subsection{Purpose and objectives}

The goal of MARS was to win the competition. To this end, the team had to design a robot capable of winning the on-site mining category of the competition. In order to win, the design needed to maximize the total mass of simulated icy regolith collected while meeting NASA's requirements. Per the Rules and Rubrics, the design of the robot had to be lightweight, efficient, autonomous, dust proof in both design and operation, and designed for operation in a simulated Martian environment (NASA, 2018). 


\section{Preliminary Design}

To begin the design process, the team generated requirements that would become the benchmarks for the design. These requirements would ensure the design met competition requirements and followed the goal of the project. The team broke the system down into functional subsystems and identified how they would interact. Then, the team generated concepts for each subsystem, scoring them against the requirements to determine the final design.

\subsection{System requirements}

The system requirements which govern the design of the robot were formed from the NASA RMC Rules and Rubrics document, as well as the goals for operation determined by the team. The key driving requirements are listed in Table 1. Driving requirements had to be attained to pass NASA requirements. Additionally, lower level requirements are listed in Table 2. Lower level requirements were set by the team as a design focus but were not required by the Rules and Rubric document.

Table 1: System Requirements

\begin{tabular}{|l|}
\hline \multicolumn{1}{|c|}{ RMC Design Requirement } \\
\hline $\begin{array}{l}\text { The robot will mine, collect, transport, and dump the regolith } \\
\text { simulant. }\end{array}$ \\
\hline The robot shall dig over $10 \mathrm{~kg}$ of regolith simulant in 10 minutes. \\
\hline $\begin{array}{l}\text { The robot shall be controllable from a remote location or } \\
\text { autonomous. }\end{array}$ \\
\hline Mass of the robot shall be under $80 \mathrm{~kg}$. \\
\hline $\begin{array}{l}\text { Energy consumption of the robot shall be recorded by } \\
\text { commercial off the shelf wattmeter. }\end{array}$ \\
\hline Dust agitation by the robot will be minimized. \\
\hline $\begin{array}{l}\text { The robot shall fit within a } 0.75 \mathrm{~m} \text { wide } \mathrm{X} 1.5 \mathrm{~m} \text { long } \mathrm{X} 0.75 \mathrm{~m} \text { high } \\
\text { area. }\end{array}$ \\
\hline The robot shall have an emergency stop button. \\
\hline The robot shall withstand at least two competition runs. \\
\hline The robot shall interface with the competition infrastructure. \\
\hline $\begin{array}{l}\text { The robot shall not use the walls or any objects outside the testing } \\
\text { area for positioning. }\end{array}$ \\
\hline $\begin{array}{l}\text { The robot shall dump the regolith into a hopper } 0.55 \mathrm{~m} \text { above the } \\
\text { BP-1. }\end{array}$ \\
\hline
\end{tabular}


Table 2: Lower Level Requirements

\begin{tabular}{|l|}
\hline \multicolumn{1}{|c|}{ Lower Level Requirements } \\
\hline Robot should be as simple as possible to improve reparability. \\
\hline $\begin{array}{l}\text { Robot should be maneuverable and overcome obstacles } \\
\text { efficiently. }\end{array}$ \\
\hline Robot should be autonomous. \\
\hline $\begin{array}{l}\text { Robot should minimize bandwidth by only transmitting needed } \\
\text { reports. }\end{array}$ \\
\hline
\end{tabular}

\subsection{Design philosophy}

The team's design philosophy focused on improving the current state of the art. Specifically, the team emphasized recreating the successes of previous competition winners while placing special focus on the optimization of digging depth, mass, and energy usage as these were identified as areas of potential improvement. Regardless of this focus, the fulfillment of competition requirements always took precedence. At design reviews, the team evaluated all mining category element projections to monitor progress.

\subsection{Target specifications}

The team established the ideal total score based on the design philosophy. These target specifications in Table 3 considered the historical performance of teams in satisfying the competition rubrics as well as the assumption performance would improve for RMC 2019. As the competition rubrics outlined points given for clearing certain objectives (passing safety inspecting, digging a given amount of gravel, autonomous performance) and also points removed based on a design's consumption of certain resources (mass, energy, etc.), the target specifications were tailored to maximize the points gained through performance and minimize the points lost from consumption.

Table 3: Target Specifications

\begin{tabular}{|l|l|l|}
\hline \multicolumn{3}{|c|}{ Target Specifications } \\
\hline Mining Element Category & Rank & Ideal (points) \\
\hline Safety Inspection & 1 & 1000 \\
\hline Gravel Dug & 2 & 135 \\
\hline Autonomy & 3 & 500 \\
\hline Robot Mass & 4 & -400 \\
\hline Energy Consumption & 5 & -25 \\
\hline Dust Tolerant Design & 6 & 30 \\
\hline Dust Free Operation & 7 & 70 \\
\hline Average Bandwidth & 8 & 0 \\
\hline Camera Bandwidth Usage & 9 & 0 \\
\hline Total & & $\mathbf{1 3 1 0}$ \\
\hline
\end{tabular}


Through the establishment and understanding of these metrics, the team was able to generate concepts and assess the completion of system requirements through tradeoff analyses, design matrices, FMEAs, and design reviews.

\subsection{Functional decomposition}

The robot assembly is a system which is decomposed into subsystems to allow for easier to manage units (Dieter, 2013). Figure 1 depicts the physical decomposition of the robot. The subsystems identified are referenced throughout this document and form the system hierarchy.

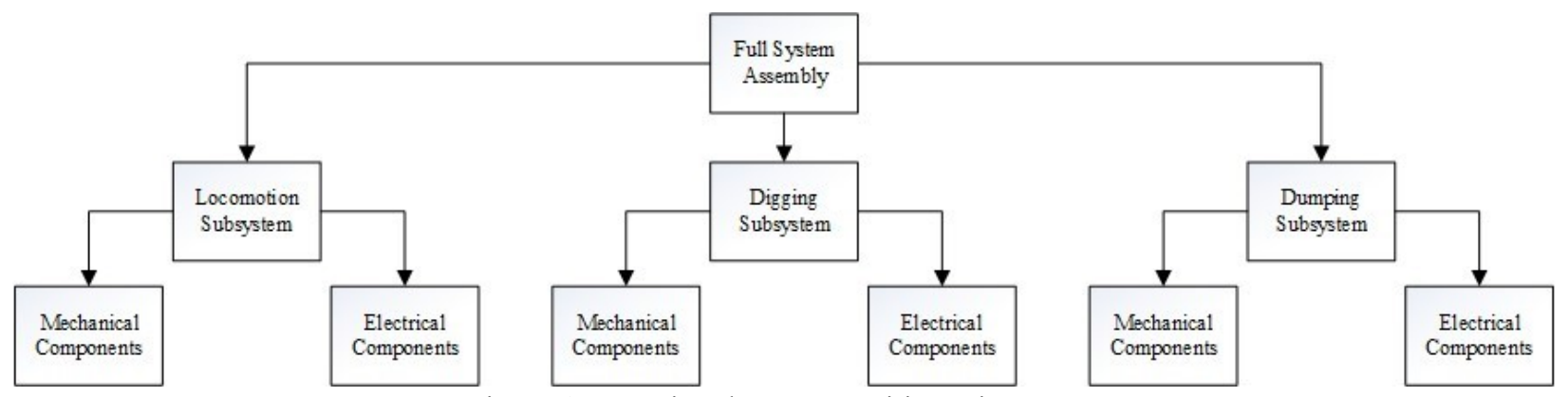

Figure 1: Functional Decomposition Diagram

\subsection{Concept of operations}

Computer vision locates a unique symbol found at the collection bin in the starting area. The robot's computer then generates a map of the working area using the established dimensions in the competition rubrics as well as the location of the collection bin as a reference point. A path is generated to reach the mining area, and this path is then executed by the locomotion system to bring the robot to the mining area. An on-board inertial measurement unit (IMU) provides feedback on the location of the robot along the path, and computer vision identifies any obstacles large enough to impede progress. Once in the mining area, the digging mechanism is actuated and begins collection. The digging mechanism feeds directly into the dumping mechanism that holds the collected regolith for transport. The onboard computer monitors the motion of the mechanism until it reaches a specified depth then retracts the digging mechanism. The locomotion system returns the robot to the starting area and positions it to dump into the collection bin. Once in position, the dumping mechanism deposits regolith into the collection bin. Another path to the mining area is generated based on the previous one, and the process repeats until the end of the ten-minute run. As the robot operates, a map of its working area and position within that area are sent to a mission control monitor, as well as information on the operation of the digging, dumping, and locomotion motors.

\section{Mechanical Design}

The mechanical design was broken up into three sub-systems: the digging, dumping, and locomotion mechanisms. The sub-system designs were based on satisfying the system requirements.

\subsection{Digging mechanism}

The design of the digging mechanism revolved around three five-inch diameter sprockets, with aluminum/carbon fiber supports. A model of the design is shown in Figure 2. The mechanical team modeled digging torques of $738 \mathrm{ft}-\mathrm{lbs}$ but it was immediately flagged as being too high. As getting a reliable theoretical value proved more difficult than expected, estimated torque values were obtained through experimental testing. The experimental torque of $4 \mathrm{ft}-\mathrm{lbs}$ was used to size the digging motor accordingly. 
From this, a drivetrain with a 112:1 gear ratio was used to give an output torque of $12.4 \mathrm{ft}-1 \mathrm{bs}$ and speed of $35.7 \mathrm{rpm}$. This drivetrain was selected to obtain the desired speed, with the advantage of excess torque. The lever arm-actuator configuration gives the belt-scoop full range to reach the bottom of the arena. Carbon fiber was used to reduce weight significantly and provide greater strength.

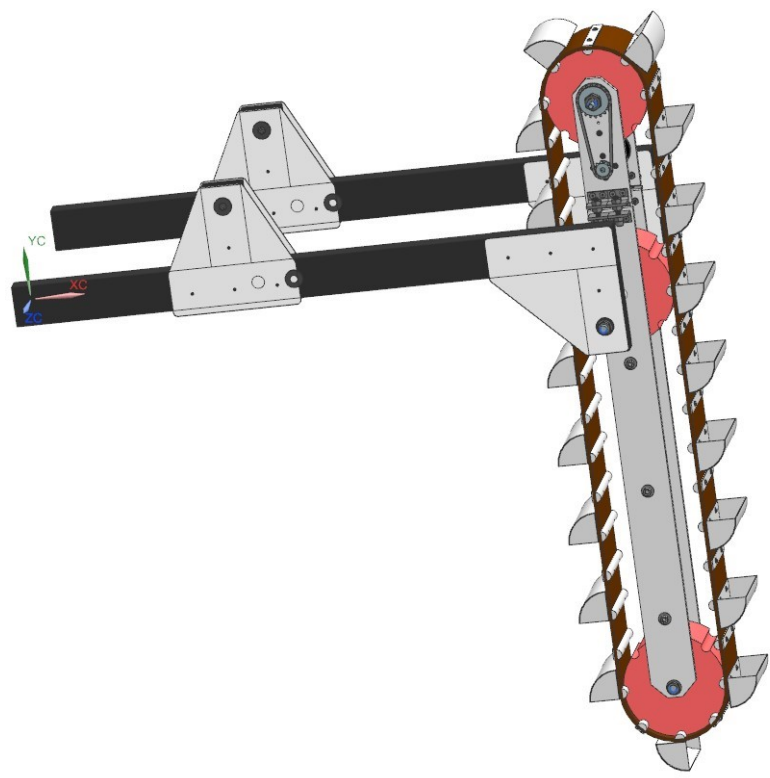

Figure 2: Digging Mechanism

\subsection{Locomotion}

The final locomotion design uses commercial off the shelf (COTS) treads with one drive motor per side, shown in Figure 3. Two aluminum plates hold steel driveshafts which connect the tensioned treads to the frame. Aluminum hex couplings are used for added torsional rigidity. A load of $80 \mathrm{~kg}$ was used to estimate the worst-case scenario required torque. With free body diagrams and basic force equations, a conservative torque estimate to move the loaded robot was calculated to be $4.6 \mathrm{ft}-\mathrm{lbs}$ per motor. A commercial system was chosen to reduce the load on the design team and provide a proven method of navigation. Completion of the locomotion system also allowed for implementation while the remaining systems were still in the design stage.

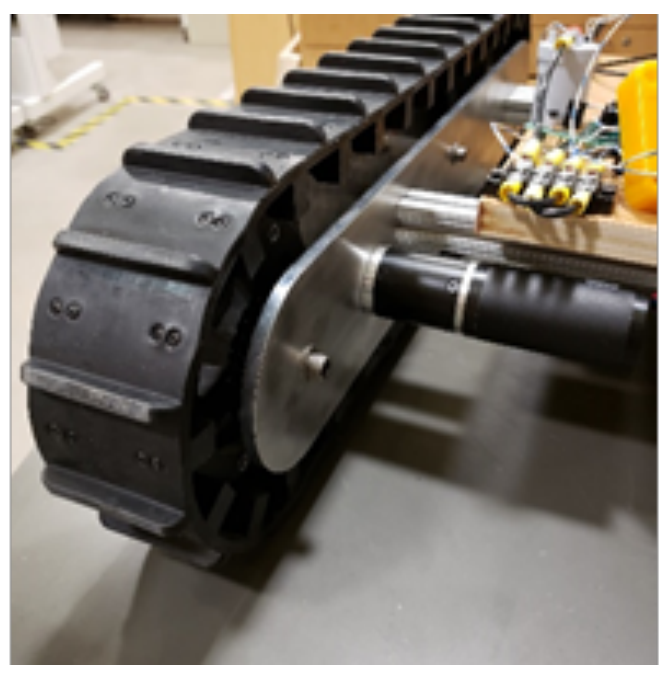

Figure 3: Assembled Locomotion Design 


\subsection{Dumping mechanism}

The dumping mechanism shown in Figure 4 was designed for simplicity. The angle of the mechanism is fixed, and a single motor drives eight idle rollers and one tensioning roller. The motor requires $3 \mathrm{ft}-\mathrm{lbs}$ of torque and rotates at $72 \mathrm{rpm}$ to ensure a quick deposit of gravel. An aluminum connecting plate attaches the motor, conveyor, and polypropylene guard to the frame. The collection area can hold an estimated $667 \mathrm{in}^{3}$. The belt is PVC plastic connected with alligator lacing. All rollers are 3D printed and supported by static titanium rods. Titanium rods give higher strength than steel while cutting the weight in half. Each roller was coated with spray paint to add friction between it and the belt. The tensioning system removes slack by using a custom threaded fastener attached to the connecting plate to extend the titanium rod during assembly. This also allowed the dumping mechanism to be adjusted to fit design changes if needed.

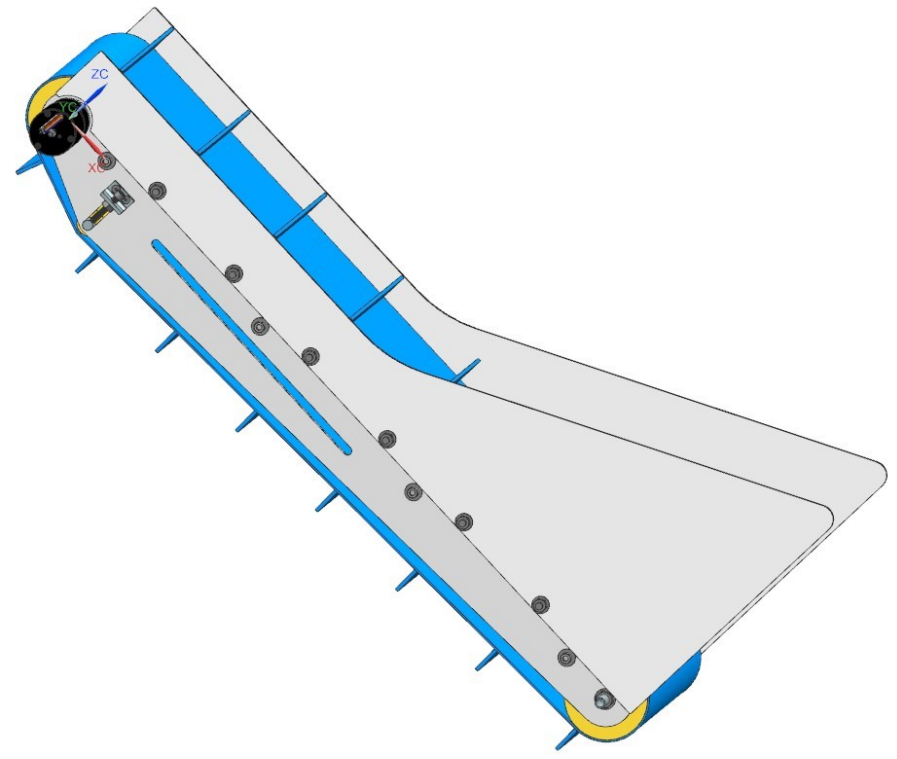

Figure 4: Dumping Mechanism

\section{Electrical Design}

The final electrical design in block diagram form is depicted in Figure 5. While several of the key components are depicted in the figure, not every component is shown for the sake of brevity.

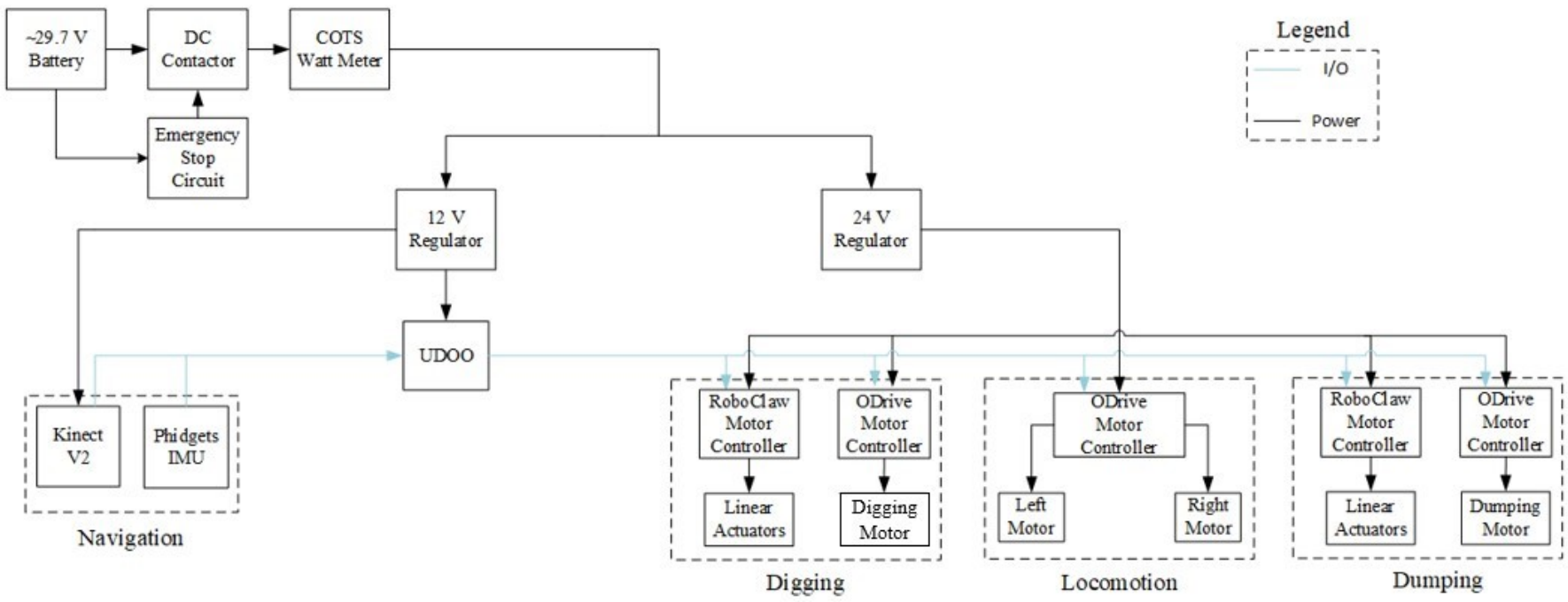


Figure 5: Electrical Block Diagram

\subsection{Power and distribution}

The robot uses two Turnigy Nano-Tech 4 Ah 4S lithium polymer batteries in series as the power source for all electronics. The power source is $29.7 \mathrm{~V}$ nominally. The team designed high efficiency buck converters to step the voltage down to the $24 \mathrm{~V}$ used by the motors and to the $12 \mathrm{~V}$ used by the control board and the relay control circuit. The team followed both the Rules and Rubrics document and ISO 13850 in selecting a larger than $40 \mathrm{~mm}$ emergency stop button (ABB, Inc). A COTS wattmeter is connected before the DC contactor in order to prevent the loss of the energy data in the event of an emergency stop.

The circuit is fused at the battery to prevent an overcurrent event from damaging the batteries. Per the RMC Rules and Rubrics, the team designed the emergency relay circuit to be isolated from the other electronics. The relay circuit is optimized using a buck converter and a voltage divider to minimize the power consumed by the circuit.

The space reserved for electronics components was limited. To minimize the electronics volume, limit mass, and improve cable management the team designed and ordered custom printed circuit boards (PCBs). PCBs were used for the buck converters as they allowed the team to use smaller surface mount components and gave the team the freedom to change the connectors used to enter the electronics box.

\subsection{Control board}

The UDOO X86 Advanced Plus was selected as the main control board for the robot as it has a $2.24 \mathrm{GHz}$ processor, an extensive supply of $5 \mathrm{~V}$ GPIO pins, and is compatible with many COTS sensors and controllers without needing a logic level converter. The UDOO X86 Advanced Plus comes with $32 \mathrm{~GB}$ eMMC storage, a standard SATA connector, a micro SD card, and an m.2 SSD slot. As weight, power consumption, and speed directly relate to the points obtained in the competition, an m.2 SSD was used. The board also comes with three USB 3.0 ports, but USB hubs were used to allow for more connections.

The key reason why the UDOO X86 Advanced Plus was selected is that it comes equipped with an Intel Curie Microcontroller. This controller allows for the UDOO to easily send input and output data and gives the team more options for interfacing. The UDOO's 6-axis combination sensor with accelerometer and gyroscope is used in conjunction with the Phidgets IMU to assist with the navigation of the robot.

\subsection{Actuators and motor controllers}

The selected actuators have primary functions in all three subsystems. It was decided that the weight of the motors was the most important feature, as weight has the highest negative affect on the amount of points in the mining portion of the competition. Continuing with this methodology, the team decided that the efficiency of the motors was the next area of consideration, as the efficiency of the motors directly correlates to the amount of energy consumed in the 10-minute run. The final highly weighted criterion was the amount of torque the motors could produce. Per the system requirements, the digging mechanism needed to produce a high torque to collect gravel.

Brushless DC motors were selected as the actuators for the locomotion, digging mechanism, and dumping mechanism. The team placed specific emphasis on mitigating the risks associated with the increased control complexity of brushless DC motors by making this decision early. The Anaheim Automation BLWRPG173S-24V-4000-R56 with attached planetary gear box was selected as the motor. The rated torque 
at the output shaft of the gearbox is $6.1 \mathrm{ft}-\mathrm{lbs}$, rotating at $71.4 \mathrm{rpm}$. To mitigate the risk associated with complexity, the team identified the $48 \mathrm{~V}$ ODrive v3.5 as the motor controller of choice. The strong community and support behind this open source project further solidified this as the brushless DC motor controller.

Linear actuators from Midwest Motion Products meeting the specifications required to adjust the height and pitch of the digging mechanism were selected. The team selected the $24 \mathrm{~V}$ variants of the LA3 linear actuator which can provide $112 \mathrm{lbs}$ of force. The selected models come equipped with feedback potentiometers to allow for position control of the linear actuators. Roboclaw $2 \times 7 \mathrm{~A}$ motor controllers were selected as a versatile and lightweight motor driver and controller for the actuators. The controller has three different interfaces allowing for flexibility in how the robot connects to the control board.

\subsection{Sensors}

For the robot to determine its position in the arena, a 3D camera and an inertial measurement unit (IMU) is used. The 3D camera is able to capture a QR code at a known location and is crucial in the startup localization process. As image processing is computationally heavy, and competition requirements prevent robots from sensing the walls of the arena, telemetry was covered primarily by two low gyroscopic drift IMUs. Rather than image processing to determine the working area, the robot uses the collection bin to orient itself and generate its working area based on the competition rubrics. Then, it determines its position within the area based on its motion relative to the starting position. Kalman filters were also implemented to minimize errors associated with long runtimes. The Phidgets Spatial Precision 3/3/3 High Resolution VINT IMU was chosen as one of the IMUs. The Phidgets IMU has a small gyroscopic drift, a low cost, and multiple Robot Operating System (ROS) packages already constructed for it. Communication between all systems occurs through ROS, which is an open-source framework for developing robotic applications. Using this IMU and the Intel Curie IMU, drift was minimized. For the 3D camera, a Microsoft Kinect V2 was used. The Kinect V2 was selected as it has a low upfront cost, a large community with ample support, and packages made for ROS. This camera also offers 70-degree horizontal field of view, a 60-degree vertical field of view, and can detect images up to 4.5 meters away.

To successfully monitor the status of each of the robot's subsystems and power consumption, current sensors were used. This detection method is used to execute autonomous procedural cues. Standard current sensors with ROS packages already created were chosen as it would be simple to integrate into the robot. The most important criteria for deciding the sensors was their rated current.

Running all applications, the max current draw was found to be $25 \mathrm{~A}$; therefore, $30 \mathrm{~A}$ rated current sensors were used to monitor the current and calculate the total power consumed. For uniformity, these same current sensors were used to measure various other subcomponents, such as motor currents.

\subsection{Software design}

The software design process was divided into three phases. The goal of the first phase was to install all the required software onto the control board. The goal second phase of the software development process was to integrate the various handlers for sensors and drivers so that the robot could be operated manually. The goal of the third stage was to develop the higher-level logic required for the robot to calibrate, navigate, and perform digging operations autonomously. 
The ROS framework allows for the construction of various nodes that can publish or subscribe to each other. ROS was chosen as the framework for this project as it has an extensive list of documentation for many components, is highly modular, and incorporates the ability to write code in Python and $\mathrm{C}++$.

The Rules and Rubrics state that communication with the robot shall be done over standard WIFI connection. This requirement allows the team to easily access the robot remotely via an SSH connection. The robot is assigned a static IP address which makes the robot easy and predictable to find on the network. After bootup, the robot runs a startup script which launches all the ROS nodes which connect the various sensors and drivers. Once connected, the controller can either run a script which starts teleoperation mode or run a script to start autonomous operation mode. In teleoperation mode the robot is controlled via the keyboard of the command computer. The display shows readouts from the IMU, current sensors, and odometry, allowing the team to view system diagnostics. Additionally, all sensors are polled at regular intervals and the values are written to a log file for analysis after each run. The manual operation mode was primarily used for testing, however during the competition run the manual operation mode was the backup for robot control in the event autonomous operation failed.

The autonomous mode of the robot utilizes the ROS package nav_core which provides functionality relating to reading in 3D point cloud sensor data, sensor filtering, position estimation, terrain mapping, setting position goals, and sending speed commands to the drive motors. These packages synergize the various components of the robot and operate as a unit.

The software uses both domain knowledge provided by the competition specifications to determine the boundaries of operation, and on the fly mapping to help orient itself and avoid obstacles that are detected. The first stage of autonomous mode is orientation, where the robot spins in place and uses its camera to find the QR codes located on the dumping bin. In the second phase, the robot enters outward navigation phase where the robot attempts to drive the shortest clear path to the digging location. The third phase is the digging phase, where the robot digs into the ground to a certain depth. The fourth phase is the return navigation, where the robot navigates back to the start area via the shortest clear path. In the fifth phase, the robot spins and locates the dumping bin once again and correct any location estimation errors. In the sixth phase, the robot backs up to the dumping bin and dump the material. The process then repeats from phase two until time is up.

The final design of the autonomous operation directly satisfies the concept of operations that the team laid out early in the design process. Several software checks were implemented to ensure safe operation of the robot. A watchdog timer on the control board is used to detect lock ups. Sanity checks of sensor data are performed to detect collisions, dig-ins, short circuits, high temperatures, and illegal actuator positions. These checks, if triggered, stop the autonomous operation of the robot immediately.

\section{Final Design}

The final design shown in Figure 6 incorporates the systems mentioned above, integrating them in a custom frame made from standard 80/20 aluminum. The final assembly also includes features for dust prevention and maintenance, discussed below. 


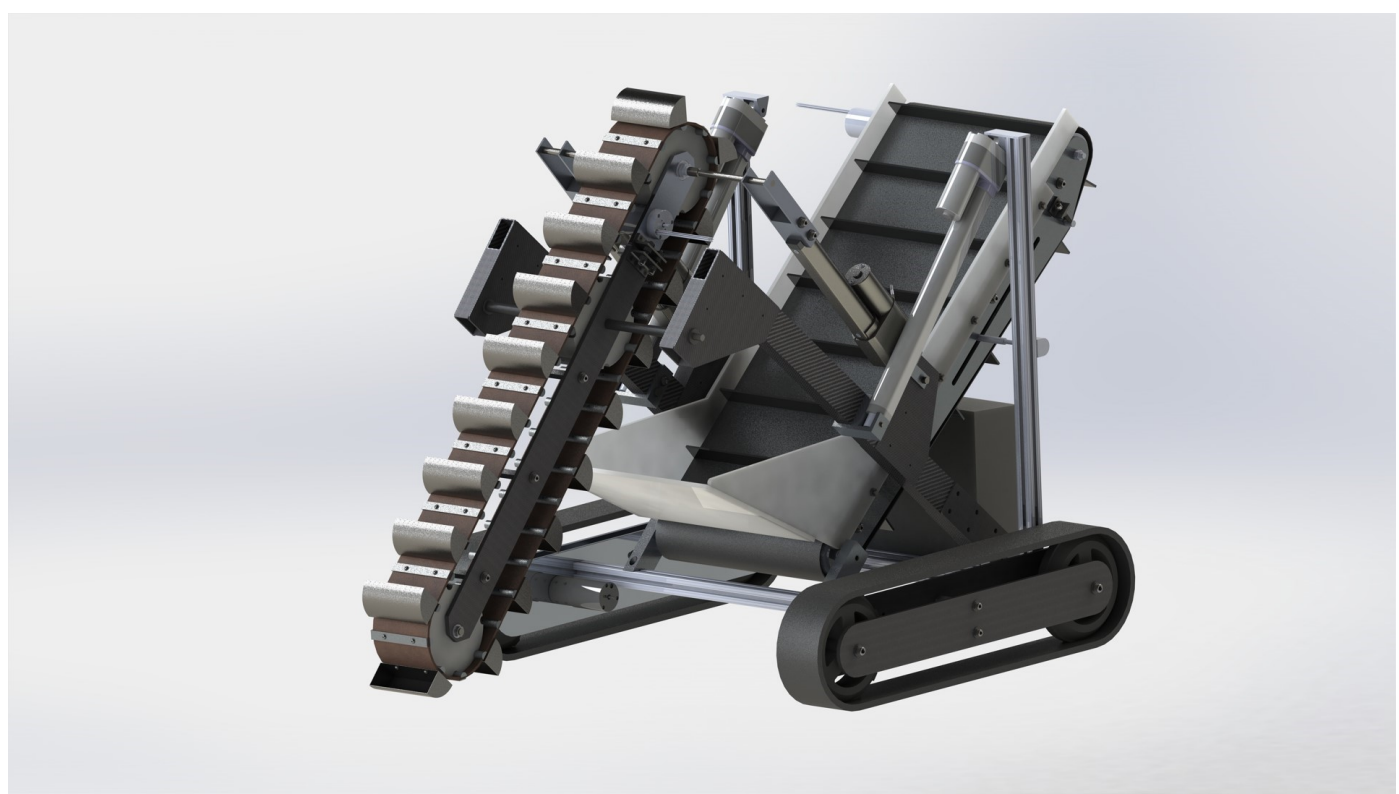

Figure 6: Final CAD Model

\subsection{Mechanical Dust Tolerance}

The subsystems were designed to have geometries which prevent the buildup of dust on surfaces. The selected linear actuators have IP65 ratings, allowing no dust ingress. The brushless DC motors were more susceptible to dust and had Frelon bushings added around the driveshafts to further reduce the chance of dust ingress, shown in Figure 7. For the digging mechanism drivetrain, a cover was 3D printed to reduce exposure of dust to the chain.

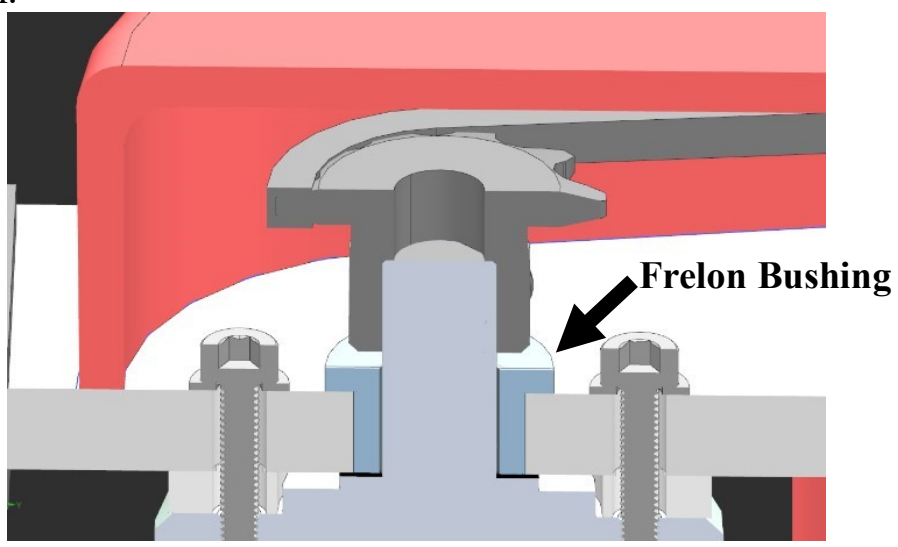

Figure 7: Frelon Bushing Sealing Motor from Environment

\subsection{Electrical Dust Tolerance}

The electronics, except for motors and the Kinect, were mounted in a sealed electronics box. While a sealed electronics box is less than ideal due to heat build-up, it is assumed that in a ten-minute competition run this heat will not adversely affect the performance of the robot. The Xbox Kinect housing was replaced with a custom 3D printed housing to protect it from dust.

\subsection{Electrical to Mechanical Interface}

The main electronics of the robot, including power distribution, control boards, and motor controllers, were housed in a plastic box at the rear of the robot. Bolted connections were used to affix the electrical box to 
the $80 / 20$ cross member. Circuit boards were mounted on insulated standoffs, and the inside of the box was coated in anti-static paint to prevent the buildup of static electricity.

Inputs and outputs at the box for both power and control wiring were made with rugged automotive connectors to ensure they were electrically and mechanically fit for the application. A connector or wire becoming disconnected during a competition run was observed to be catastrophic in previous competitions. Therefore, the team used connectors that have mechanical latches to ensure a secure connection.

All motors, linear and DC brushless, were mounted using appropriate hardware to a connecting plate ensuring a secure attachment to their respective mechanism. For non-direct-drive motors, chain and sprockets were favored over gear trains for their easy serviceability and manufacturability.

\section{Conclusion}

At the formation of this team in September 2018, MARS set out with the goal of winning the competition. The ranking of $6^{\text {th }}$ out of 50 in the competition was a respectable performance for a first-year team. The team worked on a completely new design utilizing help from industry sponsors and learned invaluable lessons about systems engineering to take with us in our careers. The completed model signifies the combination of mechanical, electrical, and computer engineering integrated into one system. The locomotion system proved to be robust enough to handle the worst obstacles encountered without failure, and the completed assembly came in under the expected weight. The largest lesson learned for the project was the importance of prioritizing tasks. Given the time constraint of the project, an expedited design of the locomotion system would allow progress for both software and manufacturing on a prototype while the remaining systems went through the design process. Another area for improvement is dust mitigation, as tension adjustments lead to dust ingress in the dumping mechanism during the competition. For future entries, the team would recommend keeping the robust locomotion platform, further developing autonomy and dust mitigation, and considering design changes to allow sifting through overburden while digging.

\section{Acknowledgements}

This project would not be successful without the support of Marquette University faculty and staff, including Thomas Silman and the Marquette Discovery Learning Center, David Newman and Opus College of Engineering Facilities, and especially the Marquette Aerospace and Robotic Systems team of Timothy

Campbell, Nathan Faust, Erik LeBeau, Konstantinos Koumoutsos, Zachary Nordgren, and Zachary Pederson. A thank you is in order once again for all of the project sponsors, including the Wisconsin Space Grant Consortium, Marquette University's Opus College of Engineering, Collins Aerospace, Komatsu Mining, Michael Campbell, and Real Chili. Thanks also to Collins Aerospace and Komatsu Mining for consultation and support of design reviews.

\section{References}

ABB, Inc. $\quad$ (n.d). Emergency $\quad$ Stops. $\quad$ Retrieved from https://library.e.abb.com/public/e32f367d17642f29852575d20068205e/1SXU150186X0201.pdf

Dieter, G., \& Schmidt, L. (2013). Engineering design (5th ed.). New York, NY: McGraw-Hill.

NASA. (2018). NASA's Tenth Rules and Rubrics. Retrieved from https://www.nasa.gov/sites/default/files/atoms/files/rmc2019_registrationrulesrubrics_09062018.pdf 